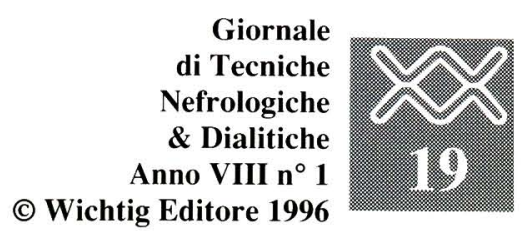

\title{
Le iponatremie
}

\author{
C. Queirolo, P. Socari \\ U.O. Nefrologia - Ospedale di Lavagna (GE)
}

e iponatremie rappresentano il più frequente squilibrio idroelettroliticio riscontrabile in clinica. Poiché il $\mathrm{Na}^{+}$e gli elettroliti di accompagnamento, i cosiddetti "anioni satelliti", sono i maggiori responsabili dell'osmolalità pl. matica, in presenza di una variazione della $\left[\mathrm{Na}_{\mathrm{p}}^{+}\right]$, deve essere valutata anche l'osmolalità. Solitamente esiste una correlazione diretta tra natremia e osmolalità plasmatica, nel senso che ad ogni variazione della natremia si associa generalmente, ma non obbligatoriamente, una variazione consensuale dell'osmolalità, tuttavia, poiché natremia non è sinonimo di osmolalità, e non esiste un'assoluta identità di comportamento tra i due valori è fondamentale precisare la differenza tra osmoregolazione e regolazione di volume:

“l'osmolalità è determinata dal RAPPORTO tra soluti $\left(\mathrm{Na}^{+}\right)$e $\mathrm{H}_{2} \mathrm{O}$; il volume dalla QUNTITÀ ASSOLUTA di sodio scambiabile $\left(\mathrm{Na}^{+} \mathrm{s}\right)$ ed $\mathrm{H}_{2} \mathrm{O}$."

L'osmolalità dipende dal "metabolismo idrico e non dalla $\left[\mathrm{Na}^{+}\right]$, quest'ultima, a sua volta, è responsabile dei "volumi" e non della osmolalità dei liquidi biologici.

Per quanto detto sarebbe più corretto sostituire il termine "iponatremie" $\mathrm{e}$ indicare con la dizione di "sindromi iponatremiche - ipoosomolari" quei quadri morbosi che, sebbene sostenuti da mec- canismi patogenetici tra loro differenti, hanno in comune:

a) dal punto di vista biochimico la ridotta $\left[\mathrm{Na}_{\mathrm{p}}^{+}\right]$

b) dal punto di vista biofisico la ridotta osmolalità plasmatica (Posm)

c) dal punto di vista clinico i sintomi della sofferenza cerebrale (nausea, vomito, torpore psichico, convulsioni, coma), muscolo tendinea (iperreflessia, normoreflessia, areflessia) e dell'apparato digerente (ileo paralitico), conseguenza della iperidratazione cellulare sistemica.

In presenza di una ridotta $\left[\mathrm{Na}_{\mathrm{p}}^{+}\right]$l'osmolalità del plasma deve essere misurata $\mathrm{e}$ calcolata. Una discrepanza di almeno 10 milliosmole $/ \mathrm{kg}$ tra i due valori, in favore della osmolalità misurata, viene indicata “GAP OSMOLALE”.

Il GAP osmolale dipende da:

\section{1) DEPLEZIONE IDRICA PLASMATICA}

Circa il 92-94\% del volume plasmatico è costituito da acqua, il restante $6-8 \%$ da lipidi e protidi. Il $\mathrm{Na}^{+}$, per la sua natura ionica, si dissolve solo nella componente acquosa del plasma. Quando si verifica un aumento della quota lipidica e/o protidica il contenuto di $\mathrm{Na}^{+}$dissolto nell'acqua plasmatica è NORMALE, ma la concentrazione misurata (fotometro a fiamma) nel volume totale risulta RIDOTTA. In questi casi si parla di PSEUDOIPONATREMIA. Nel contempo le macromolecole lipidiche e/o protidiche ben poco incidono sull'osmolalità del plasma.

2) PRESENZA NEL PLASMA DI SOLUTI A BASSO PESO MOLECOLARE

La presenza di molecole a basso peso molecolare esogene (mannitolo, glicole etilenico) o endogene (glucosio, urea) AUMENTA l'osmolalità del plasma, mentre l'effetto sulla $\left[\mathrm{Na}_{\mathrm{p}}^{+}\right]$dipende dalle caratteristiche del soluto stesso. La Figura 1 (tratta da Brenner) illustra i rapporti tra osmolalità plasmatica e natremia in presenza di sostanze diverse. Il mannitolo e il glucosio, quest'ultimo in presenza di un grave deficit insulinico, essendo molecole scarsamente permeabiidrica plasm.

osmolalità misurata $>10 \mathrm{mOsm} / \mathrm{kg}$ osmolalità calcolata $\longrightarrow$ GAP OSMOLALE 


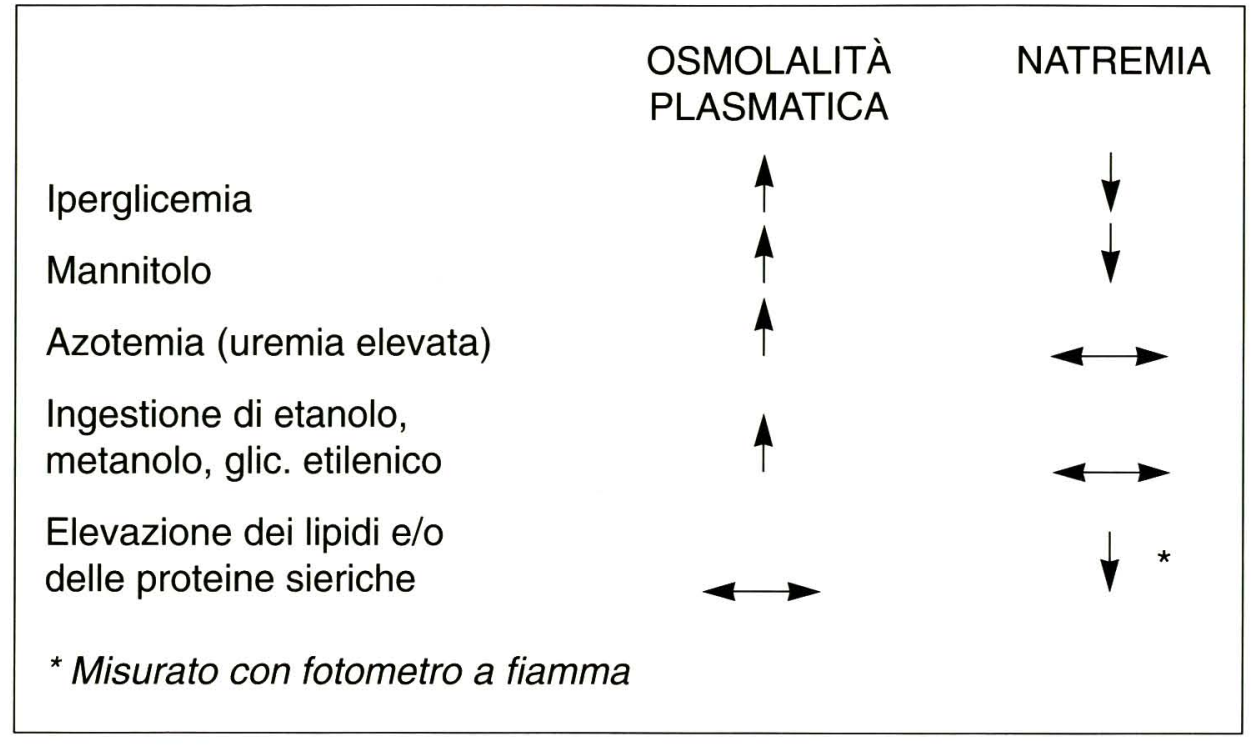

Fig. 1 - Rapporto tra osmolalità plasmatica e natremia in presenza di sostanze diverse.

li, creano un gradiente osmotico che richiama $\mathrm{H}_{2} \mathrm{O}$ dalla cellula. La conseguenza è la caduta della $\left[\mathrm{Na}^{+}\right]$. Si ha quindi una discrepanza:

Posm elevata $\longleftrightarrow\left[\mathrm{Na}_{\mathrm{p}}^{+}\right]$bassa

Al contrario il glicole etilenico, l'etanolo, il metanolo, l'urea, essendo molecole altamente permeabili, le cosiddette "osmoli inefficaci", non creano un gradiente osmotico quindi non si verifica spostamento di $\mathrm{H}_{2} \mathrm{O}$. La natremia rimane normale nonostante l'aumento dell'osmolalità plasmatica.

\section{Posm elevata $\longleftrightarrow\left[\mathrm{Na}_{\mathrm{p}}{ }_{\mathrm{p}}\right]$ normale}

Numerosi soluti intra ed extracellulari regolano l'osmolalità corporea totale ed esiste uno stretto rapporto tra:

osmolalità corporea totale $=\frac{\text { soluti extra }+ \text { soluti intra }}{\text { A.T.C. }}$

Se ci si riferisce all'extracellulare il ruolo dominante spetta al $\mathrm{Na}^{+}$, se ci si riferisce all'intracellulare questo ruolo spetta al $\mathrm{K}^{+}$, quindi la $\left[\mathrm{Na}_{\mathrm{p}}^{+}\right]$è regolata da:

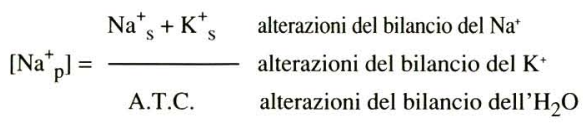

Acqua ed elettroliti circolano in rapporti fissi. Spetta al rene operare la "disgiunzione". Questo processo, conosciuto come "effetto singolo" è indispensabile per mantenere l'omeostasi idro-salina quando uno dei due elementi è in eccesso o in difetto rispetto all'altro.

La componente acquosa dell'urina è costituita da due frazioni. Una frazione rappresenta il volume di $\mathrm{H}_{2} \mathrm{O}$ necessario perché i soluti vengano escreti alla stessa osmolalità del plasma. È questa la clearance osmolale:

$\operatorname{Cosm}=\frac{\text { Uosm }}{\text { Posm }} \times \mathrm{V}_{\mathrm{u}}$

L'altra frazione, indicata come "acqua libera", libera dai soluti, rappresenta quella quota di acqua che può essere aggiunta o sottratta alla precedente frazione a seconda che l'organismo sia in eccedenza o in carenza di acqua. Questa frazione che viene indicata come Clearance dell'acqua libera può essere negativa o positiva e si calcola sottraendo al volume urinario la clearance osmolale:

$$
\mathrm{CH}_{2} \mathrm{O}=\mathrm{V}_{\mathrm{u}}-\mathrm{Cosm}
$$

Il rene è in grado di produrre urine estremamente diluite $(\sim 50 \mathrm{mosm} / \mathrm{kg})$ :

$$
\operatorname{Cosm}=\frac{50}{290} \times 100 \times 0.20=3.4 \mathrm{ml} / \mathrm{min}
$$

$$
\begin{aligned}
\mathrm{CH}_{2} \mathrm{O}=100 \times 0.20 & -3.4=16 \mathrm{ml} / \mathrm{min} \times 1440 \mathrm{~min} \\
= & \sim 23 \mathrm{litri} / \mathrm{die}
\end{aligned}
$$

È evidente la capacità, praticamente illimitata, del rene di eliminare $\mathrm{H}_{2} \mathrm{O}$ e in clinica sono eccezionali o quanto meno estremamente rari i casi di una "intossicazione idrica" da esclusivo apporto di acqua.

Perché possa manifestarsi una intossicazione idrica devono essere soddisfatte due condizioni:

\section{1) deficitaria escrezione di $\mathrm{H}_{2} \mathrm{O}$ libera \\ 2) incongruo apporto di $\mathrm{H}_{2} \mathrm{O}$ dall'esterno}

La Figura 2 suddivide i più frequenti disordini idroelettrolitici in questione in:

\begin{tabular}{l|l} 
& $\begin{array}{l}\text { A) Compromessa capacità } \\
\text { del rene di eliminare } \\
\text { quantità sufficienti di } \mathrm{H}_{2} \mathrm{O}\end{array}$ \\
IPONATREMICHE & $\begin{array}{l}\text { libera. } \\
\text { IPOOSMOLALI DA: }\end{array}$ \\
& $\begin{array}{l}\text { B) Capacità del rene di } \\
\text { eliminare notevoli quantità } \\
\text { di } \mathrm{H}_{2} \mathrm{O} \text { libera. }\end{array}$
\end{tabular}

A) Compromessa capacità del rene di eliminare quantità sufficienti di $\mathrm{H}_{2} \mathrm{O}$ libera

Perché il rene possa eliminare $\mathrm{H}_{2} \mathrm{O}$ libera devono essere soddisfatte due condizioni:

1) Integrità funzionale del tratto ascendente dell'ansa di Henle, il cosidetto "segmento diluente", sede di produzione.

2) Impermeabilità dei dotti collettori

La mancata o insufficiente escrezione di $\mathrm{H}_{2} \mathrm{O}$ libera è la conseguenza o di una ridotta produzione o di un eccessivo riassorbimento. Di frequente osservazione è la coesistenza dei due meccanismi che si potenziano reciprocamente.

Compromessa escrezione di $\mathrm{H}_{2} \mathrm{O}$ libera da aumentato riassorbimento e ridotta escrezione

a) Ipovolemia

L'iponatremia che insorge in concomitanza di uno stato ipovolemico, aggravandolo, è la risultante della coesistente permeabilità dei colettori e della ridotta produzione di $\mathrm{H}_{2} \mathrm{O}$ libera. 


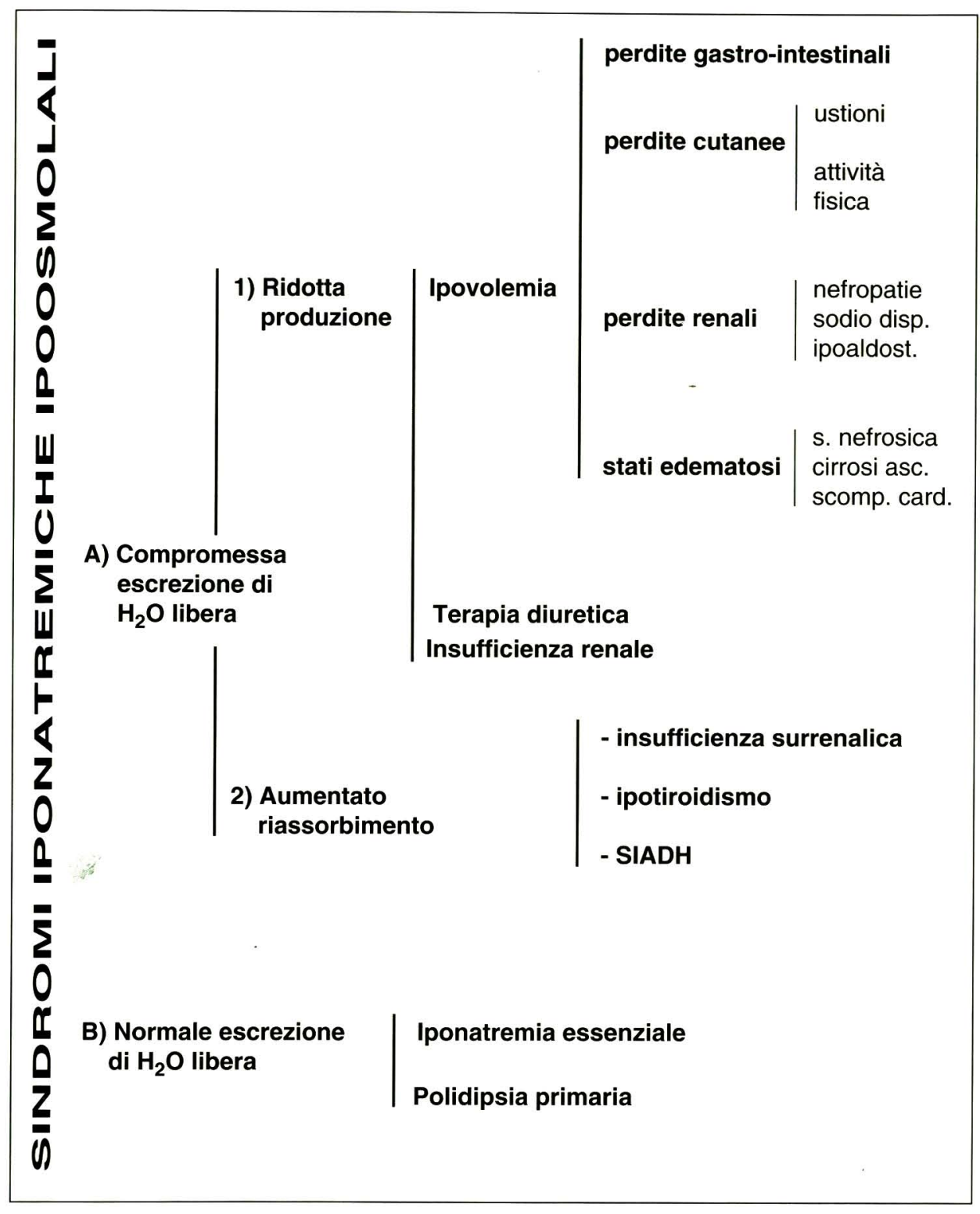

Fig. 2

- L'ipovolemia è un potente stimolo sulla secrezione di ADH. L'organismo risponde in modo appropriato alla contrazione di volume potenziando al massimo il riassorbimento. Gli stimoli volumetrici sono prioritari agli stimoli osmolali e il rene continua a trattenere acqua anche se l'osmolalità è ridotta.

- L'ipovolemia è, nel contempo, un potente stimolo sul centro della sete e l'individuo è spinto a bere indipendentemente dai valori dell'osmolalità. Trattasi sempre di una "risposta appropriata" essendo prioritario il reintegro del volume effettivo circolante. Il maratoneta va in- contro a perdite di 12 - 14 litri di liquido ipoosmotico con una concentrazione di $\mathrm{Na}^{+}$e di $\mathrm{K}^{+}$tra i 20 e i $100 \mathrm{mEq} / \mathrm{L}$. Le perdite vengono integrate con soluzioni ricche in sostanze nutrienti (carboidrati), ma estremamente povere in altri soluti. La conseguenza è la possibile insorgenza di una grava iponatremia.

Il vomito protratto e/o la diarrea spesso profusa, responsabile dello stato ipovolemico, determinano perdite isosomotiche compensate dalla assunzione di acqua e non di sali. In caso di diarrea la cospicua perdita di $\mathrm{K}^{+}$è in parete compensata dal passaggio di ioni dall'intra all'extracel- lulare; nel contempo si ha un trasferimento equimolecolare, ma in direzione opposta, di ioni $\mathrm{Na}^{+} \mathrm{e}$ ciò aggrava l'iponatremia.

- L'ipovolemia infine è responsabile di una ridotta produzione di $\mathrm{H}_{2} \mathrm{O}$ libera. La caduta del F.G. e l'intenso riassorbimento prossimale che conseguono allo stato ipovolemico riducono il flusso a livello dell'ansa di Henle, sede di formazione dell' $\mathrm{H}_{2} \mathrm{O}$ libera. L'importanza del flusso a livello dell'ansa nella produzione di $\mathrm{H}_{2} \mathrm{O}$ libera è dimostrato dall'effetto favorevole che si osserva nel cirrotico in fase ascitica in seguito a un trattamento con un diuretico osmotico (mannitolo). Il mannitolo migliora la produzione di $\mathrm{H}_{2} \mathrm{O}$ libera nel cirrotico, mentre non ha effetto nel soggetto normale.

L'azione combinata sul riassorbimento e sulla formazione di $\mathrm{H}_{2} \mathrm{O}$ libera fa sì che in caso di ipovolemia il volume dei liquidi trattenuti sia direttamente proporzionale alla quantità assunta.

La Figura 3 (tratta da Rose) sintetizza i meccanismi fisiopatologici responsabili della iponatremia nella deplezione di volume effettivo circolante.

\section{b) Terapia diuretica}

Un trattamento diuretico condotto per lungo periodo, specie se non associato a una congrua restrizione idrica può essere responsabile di una sindrome iponatremica-ipoosmolale. Sia i diuretici tiazidici che i diuretici dell'ansa possono determinare il disordine idroelettrolitico. L'ipovolemia che consegue al trattamento diuretico stimola la secrezione di ADH e il centro della sete. Se l'apporto idrico non è contenuto l'insorgenza di un quadro iponatremico - ipoosmolale è un evento frequente. Il disordine idro-elettrolitico consegue con maggior facilità al trattamento con tiazidici. Ciò dipende dal diverso punto di attacco dei due farmaci. I diuretici dell'ansa agiscono nella sede di produzione della $\mathrm{H}_{2} \mathrm{O}$ libera quindi, oltre a ridurre la produzione, limitano l'ipersomolalità dell'interstizio midollare. Il gradiente osmotico tra collettore e midollare interna è modesto e l' $\mathrm{H}_{2} \mathrm{O}$ libera prodotta viene riassorbita in limitata quantità. I tiazidici operano più a valle, non intervengono nella produzione di $\mathrm{H}_{2} \mathrm{O}$ libera, non limitano l'ipertonia della midollare. I tiazidici sono gli unici farmaci che per la loro azione, combinata con quella dell'ADH, riescono a favorire la perdita di $\mathrm{Na}^{+} \mathrm{e} \mathrm{K}^{+}$in eccesso di acqua. 


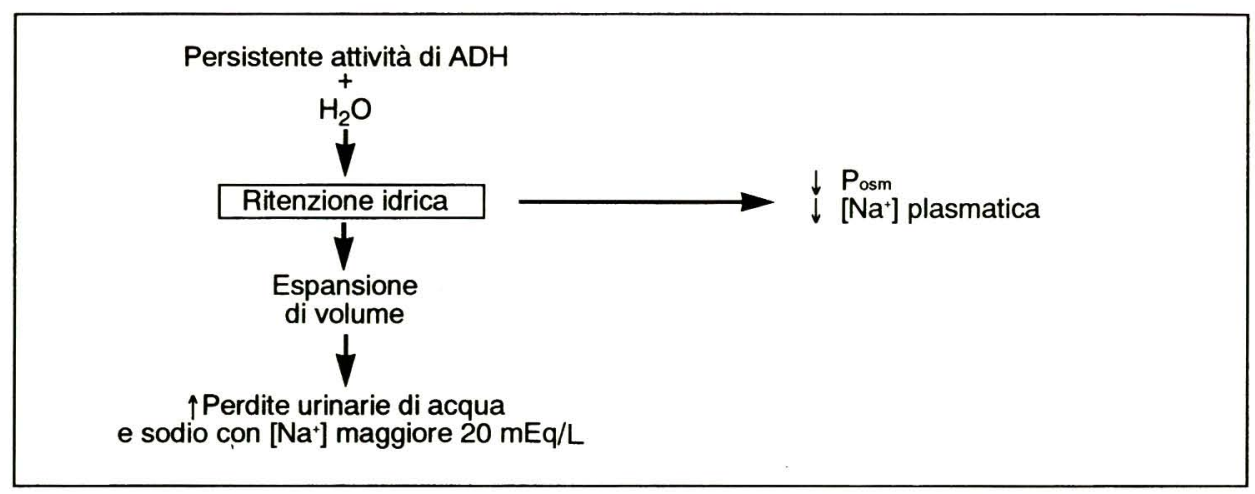

Fig. 3 - Fisiopatologia dell'iponatremia nella sindrome da inappropriata secrezione di ADH.

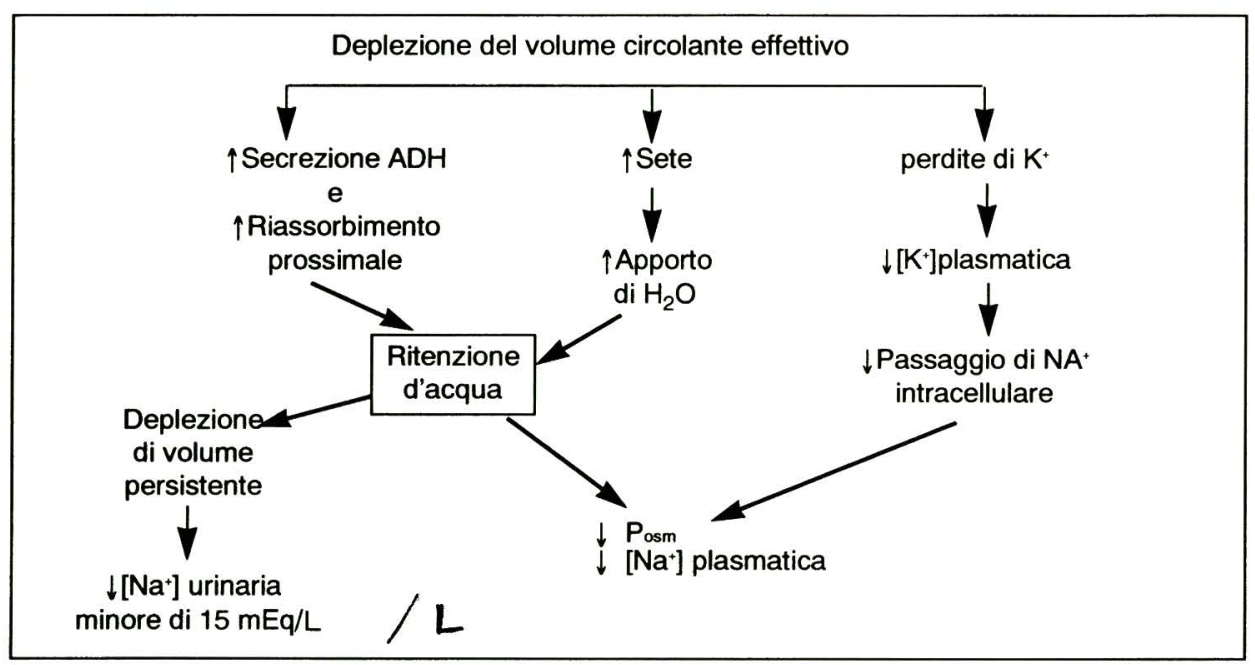

Fig. 4 - Fisiopatologia della comparsa di iponatremia nella deplezione del volume circolante effettivo.

\section{c) Insufficienza renale}

In corso di insufficienza renale cronica l'escrezione idrica, se rapportata al F.G. risulta normale per un lungo periodo e per un lungo periodo rimane normale la $\left[\mathrm{Na}_{\mathrm{p}}^{+}\right]$. Peraltro la capacità di ridurre l'osmolalità urinaria dopo carico idrico è compromessa piuttosto precocemente (diuresi osmotica). Quando il F.G. è fortemente ridotto, cade il flusso a livello dell'ansa e la capacità di produrre $\mathrm{H}_{2} \mathrm{O}$ libera viene compromessa. Se l'apporto idrico è incongruo si manifesta iponatremia.

\section{Compromessa escrezione di $\mathrm{H}_{2} \mathrm{O}$ libera da aumentato riassorbimento}

a) Insufficienza surrenalica

Il meccanismo responsabile dell'ipona- tremia in corso di insufficienza surrenalica non è completamente noto. La primitiva ipotesi che l'assenza di cortisolo rendesse i collettori maggiormente sensibili all'azione permeabilizzante dell'ADH non è stata confermata. Studi sperimentali su animali surrenalectomizzati e su tubuli collettori di animali surrenalectomizzati hanno dimostrato che $\mathrm{i}$ glicocorticoidi aumentano il turnover dell'acqua. Probabilmente sono in gioco più ordini di fattori: fattori $\mathrm{ADH}$-dipendenti e fattori $\mathrm{ADH}$-indipendenti.

1) il vomito, la diarrea, le perdite saline che caratterizzano il quadro clinico della insufficienza surrenalica sono tutti stimoli validi per attivare la ritenzione idrica. Lo stato ipovolemico opera sulla secrezione di ADH, sul centro della sete, sul F.G. e sul riassorbimento prossimale. L'effetto ultimo è l'aumento dell'apporto idrico e la ridotta produzione di $\mathrm{H}_{2} \mathrm{O}$ libera.

Secondo alcuni Autori l'ADH viene se- creto con il CRF, pertanto il cortisolo controlla la secrezione di entrambi. Questo feed-back negativo viene a mancare nell'insufficienza surrenalica e la secrezione di $\mathrm{ADH}$ non risente più di questo controllo. Il deficit prolungato di glicocorticoidi potrebbe alterare l'emodinamica intrarenale distrettuale favorendo il riassorbimento distale sganciato dall'ADH.

b) Sindrome da inappropriata secrezione di ADH (SIADH)

È il quadro paradigmatico dell'iponatremia da eccessivo riassorbimento idrico caratterizzata da:

1) dal punto di vista idroelettrolitico per l'iponatremia, la sodiuria elevata, la normalità della $\left[\mathrm{K}_{\mathrm{p}}^{+}\right]$e dei $\left[\mathrm{HCO}_{3}^{-} \mathrm{p}\right.$;

2) dal punto di vista biofisico per l'ipoosmolalità plasmatica e l'ipersomolalità urinaria;

3) dal punto di vista dell'equilibrio acido-base la normalità del $\mathrm{pH}$ plasmatico;

4) dal punto di vista clinico per la mancanza di edemi, l'espansione dei volumi, la normalità della funzione renale, surrenalica e del $\mathrm{pH}$.

La secrezione di $\mathrm{ADH}$ è totalmente o parzialmente sganciata dagli stimoli fisiologici in particolare dagli stimoli osmocettoriali. La consegueza è il persistente riassorbimento della $\mathrm{H}_{2} \mathrm{O}$ libera prodotta.

Nessuno stimolo fisiologico (osmotico, volumetrico) giustifica il riassorbimento idrico che, per questa ragione, viene definito "inappropriato". L'escrezione di $\mathrm{Na}^{+}$è normale perché l'espansione di volume, conseguenza della ritenzione idrica, operando sui volocettori condiziona una appropriata sodiuria e giustifica la mancata formazione di edemi.

La Figura 4 (tratta da Rose) sintetizza i meccanismi fisiopatologici responsabili dell'iponatremia in corso di SIADH.

Il quadro è dominato dalla ritenzione idrica; se l'apporto idrico viene fortemente ridotto, si raggiunge un "nuovo equilibrio" caratterizzato dalla STABILITÀ della ridotta $\left[\mathrm{Na}_{\mathrm{p}}^{+}\right]$senza altri disturbi. La persistenza dell'iponatremia è la conseguenza della natriuria $(>20$ $\mathrm{mEq} / \mathrm{L}$ ).

Sono stati individuati 4 modelli di SIADH.

Il primo modello è paradigmatico delle sindromi iponatremiche-ipoosmolali da 
incontrollato riassorbimento di $\mathrm{H}_{2} \mathrm{O}$ libera. La secrezione di $\mathrm{ADH}$ è sganciata da ogni stimolo físiologico o vi è una produzione ectopica e incontrollata di ormone.

Il secondo modello è paradigmatico di quei quadri che insorgono nonostante il rene sia in grado di eliminare 1 ' $\mathrm{H}_{2} \mathrm{O}$ prodotta.

Il terzo modello si caratterizza per una "risposta parziale" agli stimoli osmolali. È come se una frazione di ADH fosse insopprimibile.

Il quarto modello si caratterizza per la sensibilità all'ADH che gli organi bersaglio di alcuni soggetti dimostrano in particolari condizioni.

B) Sindromi iponatremiche - ipoosmolali con normale escrezione di $\mathrm{CH}_{2} \mathrm{O}$

\section{1) Iponatremia essenziale}

Gli osmocettori sono strutture estremamente sensibili alle variazioni dell'osmolalità plasmatica. In soggetti molto sensibili, variazioni di $0.5 \mathrm{mOsm} / \mathrm{kg}$ sono suff ienti a determinare risposte appropriate. Al di sotto dei valori soglia (275 mOsm $/ \mathrm{kg}$ ) la concentrazione plasmatica di $\mathrm{ADH}$ non è rilevabile, al di sopra dei valori soglia $(290 \mathrm{mOsm} / \mathrm{kg})$ la concentrazione ormonale aumenta in maniera direttamente proporzionale all'osmolalità. In corso di iponatremia essenziale la $\left[\mathrm{Na}_{\mathrm{p}}^{+}\right]$è stabile intorno a valori di 125 - $130 \mathrm{mEq} / \mathrm{L}$, ma la capacità che hanno questi soggetti di eliminare rapidamente un carico idrico indica che $\mathrm{i}$ recettori sono funzionanti, rispondono in modo adeguato, ma sono tarati per valori di osmolalità molto più bassi. (< 260 $\mathrm{mOsm} / \mathrm{kg}$ ).

Il quadro ritenuto un tempo estremamente raro, in realtà forse è più frequente anche di quanto si creda oggi. La difficoltà diagnostica risiede nella riluttanza di sottoporre a un carico idrico un soggetto che presenta una $\left[\mathrm{Na}^{+}\right]$stabile, oscillante tra i $125-130 \mathrm{mEq} / \mathrm{L}$.

\section{2) Polidipsia primaria}

L'apporto idrico, anche se estremamente elevato, viene normalmente eliminato e la $\left[\mathrm{Na}_{\mathrm{p}}^{+}\right]$risulta normale o solo modestamente ridotta. In particoli soggetti (patologia psichica sotto terapie specifiche) l'apporto idrico può superare la capacità escretrice del rene.

\section{BIBLIOGRAFIA}

1. Ahmed AB, George BC, Dingman JF. J Clin Invest 1967; 46: 111.

2. Albrink MJ, Hald PM, Man EB, Peters JP. J Clin Invest 1955; 34: 1481.

3. Anderson RJ, Cadnapaphornchai P, Arbdttle JA. J Clin Invest 1974; 54: 1473.

4. Anderson RJ, Chung HM, Kluger R, Schrier RW. Ann Intern Med 1985; 102: 164.

5. Arieff AL. N Engl J Med 1986; 314: 1529.

6. Arieff AL, Llach F, Massry SG. Medicine 1976; 55: 121.

7. Ashouri OS. Arch Intern Med 1986; 146: 1355.

8. Ashraf N, Loksley R, Arieff AL. Am J Med.

9. Bartoli E. "Fisiopatologia e clinica degli squilibri idroelettrolitici" da: Medicina Internazionale n. 23, Novembre 1975.

10. Bartter FC, Schwartz WB. Am J Med 1967; 42: 790.

11. Brenner BM, Rector FC. The Kidney 1991, Sanders Company. Philadelphia.

12. Collins RD. Manuale illustrato degli squilibri idroelettrolitici. Il pensiero Scientifico Editore. 1978.

13. Edelman IS, Leibman J, O' Meara MP, Birkenfel DL. J Clin Invest 1958; 37: 1236.

14. Frizzel RT, Lang GH, Lowance DL, Lathan SR. J Am Med Assoc 1985; 255: 772 .

15. Gill JR, Gann DS, Bartter FC. J Clin Invest 1962; 41: 1078.

16. Kinsey S. Il ricambio idroelettrolitico. Il pensiero Scientifico Editore. 1982.
17. Laragh JH. J Clin Invest 1954; 33: 807.

18. Linas SL, Bert T, Robertson GL. Kidney Int 1980; 18: 58.

19. Maschio G. Il metabolismo elettrolitico e minerale nelle malattie del rene. Piccin Edit. 1980.

20. Petrella E. Le basi fisiopatologiche degli squilibri idroelettrolitici e acido base. Mc Graw-Hill Ed. 1990.

21. Rose BD. Am J Med 1986; 81: 1033.

22. Rose DB. Fisiologia clinica dell'equilibrio acido base e dei disordini elettrolitici. Mc Graw-Hill Ed. 1991.

23. Schmale H, Fehr S, Richter D. Kidney Int 1987; 32: 8.

24. Verbalis JG. Am J Physiol 1984; 247: E540.

25. Wolfson B, Manning RW, Davis LG. Nature 1985; 315: 59. 